\title{
REVISTA
}

Revista Educación

ISSN: 0379-7082

ISSN: 2215-2644

revedu@gmail.com

Universidad de Costa Rica

Costa Rica

\section{Observando la educación: Tramas y desafíos desde la investigación situada}

\author{
Caldwell-Bermúdez, Clyde; Camacho-Calvo, Silvia; Díaz-Soucy, Cecilia; González-Zúñiga, Marianela; \\ Hilje-Matamoros, Walter; Monge-Rodríguez, Melania; Muñoz-Varela, Luis; Vargas-Sandoval, Yensi \\ Observando la educación: Tramas y desafíos desde la investigación situada \\ Revista Educación, vol. 41, núm. 2, 2017 \\ Universidad de Costa Rica, Costa Rica \\ Disponible en: http://www.redalyc.org/articulo.oa?id=44051357013 \\ DOI: http://dx.doi.org/10.15517/revedu.v41i2.27186
}

Esta obra está bajo una Licencia Creative Commons Atribución-NoComercial-SinDerivar 3.0 Internacional. 


\section{Observando la educación: Tramas y desafíos desde la investigación situada}

\section{Observing Education: From the Situated Research Threads and Challenges}

Clyde Caldwell-Bermúdez

Universidad de Costa Rica, Costa Rica

clyde.caldwell@ucr.ac.cr

Silvia Camacho-Calvo

Universidad de Costa Rica, Costa Rica

silvia.camachocalvo@ucr.ac.cr

Cecilia Díaz-Soucy

Universidad de Costa Rica, Costa Rica

mariacecilia.diaz@ucr.ac.cr

Marianela González-Zúñiga

Universidad de Costa Rica, Costa Rica

marianela.gonzalez@ucr.ac.cr

Walter Hilje-Matamoros

Universidad de Costa Rica, Costa Rica

walter.hiljematamoros@ucr.ac.cr

Melania Monge-Rodríguez

Universidad de Costa Rica, Costa Rica

melania.monge@ucr.ac.cr

Luis Muñoz-Varela

Universidad de Costa Rica, Costa Rica

luis.munoz@ucr.ac.cr

Yensi Vargas-Sandoval

Universidad de Costa Rica, Costa Rica

yensi.vargas@ucr.ac.cr
DOI: http://dx.doi.org/10.15517/revedu.v41i2.27186

Redalyc: http://www.redalyc.org/articulo.oa?id=44051357013
Recepción: 05 Diciembre 2016

Aprobación: 22 Mayo 2017

\section{Resumen:}

El artículo tiene por finalidad exponer aspectos del quehacer de investigación que se lleva a cabo en el Observatorio de la Educación Nacional y Regional (OBSED)- del Instituto de Investigación en Educación (INIE). Se hace un breve análisis acerca de las políticas públicas en educación adoptadas durante los últimos años por los países de Centroamérica, marcadas especialmente por las políticas del Banco Mundial y de la UNESCO. El análisis conduce, en particular, a reflexionar sobre la política educativa costarricense, situándola en el contexto de los perfiles laborales que demanda el mercado de trabajo vinculado a la globalización económica, así como en relación con las desigualdades sociales estructurales, la inequidad, y el incremento de la pobreza que se registra en los países de la región centroamericana. Se aborda también el análisis de dos aspectos centrales que determinan las políticas públicas en educación: los enfoques y estrategias de evaluación de los procesos y los sistemas educativos y la dimensión de la interculturalidad como una necesidad epistémica/pedagógica insoslayable, que debe ser incorporada en los procesos educativos, a fin de avanzar en atender y resolver las situaciones de la inequidad, la desigualdad y la exclusión educativa estructural. Se tiene por finalidad poner en perspectiva la necesidad de una educación que reconozca y releve las necesidades y expectativas de las personas y las comunidades situadas en sus respectivas realidades socioculturales, en relación con el análisis y la reflexión acerca de los procesos socio/educativos en el país y en América Latina y el Caribe.

Palabras Clave: Políticas educativas, globalización económica, integración regional, empleabilidad, evaluación transformadora, interculturalidad, colonialidad.

\section{ABstract:}


The article aims to expose aspects of the research carried out in the Observatory of National and Regional Education -OBSED- of the INIE. A brief analysis is made of the public policies in education adopted in recent years by the countries of Central America, especially marked by the policies of the World Bank and UNESCO. The analysis leads in particular to reflect on the Costa Rican educational policy, placing it in the context of job profiles demanded by the job market linked to economic globalization, as well as in relation with structural social inequalities, inequity, and the increase of poverty registered in the countries of the region. It also addresses two central aspects that determine education public policies: the approaches and strategies of educational systems and the dimension of interculturality as an unavoidable epistemic/pedagogical need, which must be properly incorporated in the educational processes in order to advance in addressing, and resolving situations of inequity, inequality and structural educational exclusion. The final purpose is to put into perspective the need for an education that recognizes and relieves the needs and expectations of people and communities located in their respective socio-cultural realities, in relation to the analysis and reflection on socio-educational processes in the Country and in Latin America and the Caribbean.

KEYWORDS: Education policies, economic globalization, regional integration, employability, evaluation, interculturality, coloniality.

\section{INTRODUCCIÓN}

En el marco de la celebración del sesenta aniversario de la Facultad de Educación de la Universidad de Costa Rica y del quinto aniversario de la creación del programa de investigación Observatorio de la Educación Nacional y Regional -OBSED- del Instituto de Investigación en Educación -INIE-, se dispone plasmar, de manera colaborativa, en el presente texto, una parte de los resultados y los esfuerzos del análisis e intercambio que se llevan a cabo en este programa, con la finalidad de generar aportes a la discusión y a la reflexión acerca de la realidad educativa actual.

El OBSED, creado en 2012, nace a raíz del proceso reflexivo llevado a cabo por el Instituto de Investigación en Educación -INIE-, en el marco de la formulación del Plan Estratégico 2009-2014. Según se señala en el Plan, en relación con el eje de gestión estratégica, la agenda de investigación y de trabajo del INIE debe responder, entre otras finalidades: a) a estudiar y atender con resultados de investigación y de proyectos de acción social, las necesidades de información y de capacitación de las diversas comunidades del país; b) vincularse con instituciones públicas y privadas de índole nacional e internacional; c) proponer y facilitar propuestas de solución y de transformación que contribuyan a la construcción de una sociedad justa y solidaria.

Se planteó, así, la creación de un programa de investigación que incluyera, como sus principales componentes, la apertura de espacios para la reflexión y el análisis del estado de la educación costarricense, con posibilidades de ampliar su mirada hacia Centroamérica y el Caribe. Se dispuso también desarrollar un espacio virtual, por medio del cual se pretende facilitar información y abrir espacios de diálogo, reflexión y debate acerca de la realidad educativa nacional y regional, así como proyectar una mirada sobre la educación en toda su complejidad y riqueza.

Es así como se logra la consolidación de un equipo investigador que buscó abrir nuevos caminos de articulación para ampliar la participación de todos los sectores de la sociedad civil interesados en la educación, sus implicaciones y desafíos.

Se han llevado a cabo actividades académicas de distinta índole, tales como: talleres, conversatorios y foros, que han permitido informar, comprender, dialogar, discutir y crear opinión en torno a acontecimientos y tópicos educativos concernientes a la realidad nacional y regional. Estas actividades han tenido como eje el abordaje del análisis, la discusión y el debate en torno al estado de la cuestión educativa, así como respecto de los desafíos que, en el campo educativo, hoy se presentan diversos y de significado trascendente para el presente y futuro de la sociedad costarricense y de los países de la región centroamericana.

Se ha abordado con especial énfasis el estudio de las políticas públicas en educación, en temáticas como oferta educativa, programas y estrategias para la atención a la inequidad, la desigualdad y la exclusión, la evaluación de programas de atención especial focalizada, las nuevas propuestas de formación educativa 
basadas en la educación técnica para la empleabilidad (educación por competencias o educación dual, por ejemplo) y otras diversas temáticas más.

Estos espacios y formas de proyección del OBSED le han dado un gran dinamismo al quehacer investigativo; a su forma de mirarlo y de abordarlo. Su equipo investigador se caracteriza por una posición de sujeto colectivo cognoscente crítico, propositivo y sensible a los cambios, el cual lleva a cabo periódicamente una reflexión epistemológica acerca de las interrogantes que emergen en la relación con procesos, sujetos, acontecimientos, aproximaciones metodológicas actualizadas, que articulen la excelencia de la investigación con las variadas formas de conocimiento que cada persona investigadora desarrolla, fundamentándolas ante los hallazgos que producen los resultados de la investigación inter y transdisciplinar.

Mediante esta perspectiva epistémica de mirar, comprender y llevar a cabo la investigación, el OBSED busca una aproximación efectiva hacia la construcción social del conocimiento y la comprensión de la realidad social, política y educativa en la que nos desenvolvemos.

En el presente artículo se hace exposición de algunos de los enunciados programáticos que reflejan las tramas investigativas en las que actualmente el equipo de investigación del OBSED se encuentra involucrado y que tienen por finalidad realizar análisis crítico y propositivo acerca de la realidad educativa nacional y regional.

\section{Políticas educativas: Aproximación a la región Centroamericana}

Dentro de los determinantes de la política educativa centroamericana de las últimas dos décadas, las propuestas marco para los países de la región las han construido, en gran medida, los organismos internacionales como el Banco Mundial (BM) y la Organización de las Naciones Unidas para la Educación, la Ciencia y la Cultura (UNESCO).

En materia de políticas económicas, especialmente las propuestas del BM han tenido fuertes impactos, no solo en el ámbito educativo sino también en lo social, con consecuencias significativas para los países de la región.

Observar la incidencia de los acelerados cambios sociales vividos desde finales del siglo XX ayuda a ubicar en qué contexto se toma la decisión de elaborar las agendas internacionales; este entorno ha forzado a reconfigurar los ámbitos organizativos de lo social, político, económico, educativo y cultural. Se adiciona también la utilización de la tecnología de la información y la comunicación, que ha venido a mediar y a apresurar los cambios relacionados con el desarrollo de la región.

Brunner, citado por Valerión y Esquivel (2006), señala que esa reorganización del espacio no es solo del mercado en términos de economía: "El reordenamiento social y cultural que tales cambios implican supone, desde la perspectiva de la justicia y la equidad, la participación de todos los seres humanos como ciudadanos y ciudadanas responsables que optan por las opciones que aseguren un desarrollo social y económico integral, sustentable y sostenible" (p. 2).

Los cambios acontecidos a partir de los años sesenta y hasta las últimas décadas del siglo XX en las economías de las principales potencias acarrearon transformaciones sociales de alto impacto que vinieron a acrecentar las brechas de la desigualdad estructural en los países y entre estos mismos. Este escenario colocó la educación como un componente fundamental para desarrollar el capital humano y las destrezas y capacidades de productividad de la población, que ahora requieren también la complementación de la tecnología para adaptarse a los cambios económicos y sociales acaecidos últimamente.

No obstante, en los países de la región centroamericana y en general de América Latina, el cambio tecnológico, lo mismo que la adecuación formativa del capital humano no alcanza a resolver las situaciones prevalecientes de la desigualdad social estructural. En su lugar, más bien se produce un incremento de la pobreza y la inequidad, entretanto que también acontece, como contrapunto, y cada vez en menos manos, una mayor concentración de la riqueza. 
El Banco Mundial, como organismo financiero multilateral de alta influencia en los países, ha direccionado sus políticas y recursos hacia la transformación de los sistemas educativos, con el fin de que cada país responda a las demandas que la economía global establece. La posibilidad de realizar dicha transformación será viable, si se forma y capacita a la población y a las nuevas generaciones en educación formal, no formal y técnica, para atender la demanda de perfiles laborales que establece el mercado de trabajo definido en perspectiva de globalización económica. El BM ha remarcado dentro de sus políticas que los países lleven a cabo acciones para mejorar la eficiencia del sector educativo y focalizar los recursos y políticas para los niveles primarios de la educación. Todo ello, desde una perspectiva de mercado, según los modelos económicos emergentes.

La UNESCO, por su parte, ha convocado a las naciones a asumir compromisos concertados a escala internacional, por medio de conferencias y foros en que se ha recalcado el derecho a la educación como un derecho humano y la necesidad de universalizar el acceso y la cobertura educativa: Conferencia de Jomtien (1990), Marco de Acción de Dakar (2000); los Objetivos de Desarrollo del Milenio -ODM-, vigentes hasta 2015; así como los Objetivos del Desarrollo Sostenible -ODS-, propuestos con metas para su cumplimiento al año 2030.

Se propone que estos acuerdos mundiales sean asumidos por los países e incorporados en la formulación de las políticas educativas, haciendo énfasis en garantías universales como el acceso y la cobertura, priorizando la atención a los sectores sociales vulnerables y brindando una educación efectiva y de calidad. El carácter universalista de las declaraciones marca la pauta para que los países las adopten y las implementen.

La cobertura educativa, fundamentalmente en primaria, es una de las prioridades, pero también lo son las etapas iniciales a ella y la secundaria. Para la región centroamericana, erradicar el analfabetismo sigue siendo una prioridad. La posición de la UNESCO subraya que la elaboración de políticas públicas es sumamente importante para optimizar los recursos destinados a la educación y su adecuada distribución, lo que representa un gasto moderado sobre los dineros destinados al gasto público de cada país. Valora a la educación como un medio para reducir la desigualdad en los países de la región. Propone elaborar políticas educativas que garanticen mayores oportunidades de educación para la población. Construir políticas basadas en el componente de la equidad (González, 2014). Tomar en cuenta no solo el acceso y la cobertura, sino también la permanencia en el sistema educativo de niños, niñas y jóvenes, sin dejar de lado la calidad de la enseñanza y el aprendizaje.

La calidad educativa representa un desafío y una tarea urgente, asociada a los cambios acelerados de las sociedades en la región. Esto implica también elaborar políticas dirigidas a la mejora de la formación profesional en educación, de manera tal que el proceso educativo pueda ser efectivamente aprovechado por las poblaciones estudiantiles y que la formación recibida contribuya a la mejora de las condiciones sociales de vida.

Las diversas reuniones que organizan los organismos multilaterales contribuyen a acordar consensos entre los países y a conformar equipos de trabajo, como es el caso del Proyecto Regional de Educación para América Latina y el Caribe \# PRELAC \#, que pretende ver de una manera holística la cuestión educativa, desde el desarrollo integral de la población estudiantil, la diversidad sociocultural y la educación como un tema que debe involucrar a todas las personas que habitan un país.

En Centroamérica se sistematizan los aportes de los organismos para elaborar propuestas de política educativa. La Coordinadora Educativa y Cultural Centroamericana -CECC- institución que pertenece al Sistema de la Integración Centroamericana -SICA-, es el organismo que tiene a su cargo la integración y cooperación regional en materia de educación y la cultura. La CECC define la Política Educativa Centroamericana -PEC- como "el conjunto de orientaciones para dotar a la región centroamericana de un marco general de acción en materia educativa, de acuerdo con las prioridades regionales identificadas" (Coordinación Educativa y Cultural Centroamericana; Sistema de la Integración Centroamericana (CECC/SICA), 2013, p. 2). 
A pesar de la recurrente crisis económica de los últimos años, los países centroamericanos han realizado esfuerzos por aumentar la inversión en educación, vista por los gobiernos de manera prioritaria y como estrategia para el desarrollo económico y social. Este aspecto, no obstante, presenta resultados dispares entre los países de la región y plantea profundizar el análisis de los sistemas y los procesos educativos de cada país, para identificar cuáles aspectos son vinculantes con la educación y por qué no se subsanan las necesidades de cobertura, calidad y pertinencia de la oferta educativa.

\section{Costa Rica: La Política EdUCATIVA NACIONAL EN CONTEXTO}

Según se expone en el texto de la Política Educativa hacia el Siglo XXI, aprobada por el Consejo Superior de Educación de Costa Rica en 1994 y vigente al día de hoy, las transformaciones estructurales que acontecen en el mundo a partir de las décadas de 1980 y 1990 plantean la necesidad de llevar a cabo una reorganización institucional y programática del Estado costarricense, para poder atender el "cambio de paradigma que se ha dado a finales del siglo XX”. En la óptica de la Política Educativa:

Esto implica transformar las concepciones de desarrollo desde una perspectiva, deshumanizada y deshumanizante, del uso
irracional de los recursos naturales, del desarrollo tecnológico como un fin en sí mismo, del incremento del capital físico como
única fuente de riqueza y de una estrategia de desarrollo y competencia internacional con base, simplemente, en la estructura
de mercado, hasta una concepción espiritual-humanista y humanizadora, de sostenibilidad de los recursos naturales, de la
tecnología al servicio de la persona, del incremento y mejoramiento en las habilidades y destrezas y una estrategia de desarrollo
centrada en cada ser humano del país. (Consejo Superior de Educación, 1994, párr. 9)

La nueva situación estructural de "cambio de paradigma", según queda enunciada en la referencia anterior, la Política Educativa hacia el Siglo XXI la enmarca en el concepto de "sostenibilidad", instituyendo a este como la referencialidad básica en la que se ha de sustentar la orientación y vertebración de la política educativa nacional, los programas especiales de atención focalizada a los sectores estudiantiles en condición de vulnerabilidad socioeconómica, así como la oferta educativa que conforman el sistema y el proyecto educativo del país.

La "Política" resalta cinco áreas básicas, como las vertientes en las que ha de articularse la política educativa y el proyecto educativo del país. De conformidad con los requerimientos a atender para dar curso a la perspectiva de la "sostenibilidad", dichas cinco áreas son las siguientes: a) ambiental, b) recurso humano, c) social y política, d) económica y productiva; e) ética.

En Costa Rica, desde hace al menos un cuarto de siglo, se adoptó una nueva matriz de política pública para organizar de manera estructural el aparato económico/productivo: el esquema de la atracción de inversión extranjera directa, instalación de empresas transnacionales dedicadas a la industria de alta tecnología, plantas de producción industrial para la exportación en régimen de zonas francas, desarrollo intensivo del turismo y del sector terciario (servicios), fomento a las exportaciones y apertura de la economía nacional al mercado internacional.

El nuevo modelo de crecimiento se basó en la apertura económica. Esto significaba una menor participación del Estado en las decisiones económicas, dejando que el sector privado asumiera mayores riesgos. Esta apertura implicaba, además, ver "hacia afuera", en lugar de enfocarse tanto en el mercado interno. Los productores nacionales deberían, de ese momento en adelante, competir con los internacionales. (Mesalles, 2011, p. 68)

Esta nueva situación produjo, como consecuencia, que las actividades productivas en diversas comunidades y regiones geográficas del país pasaran a quedar inscritas en una lógica de agresiva competencia impuesta por la colocación en el mercado nacional de productos provenientes del extranjero (el desmantelamiento de la producción de frijoles en la Región Huetar Norte, por ejemplo), al tiempo que también institucional y estatalmente desprotegidas. Una diversidad de actividades productivas en el sector agrícola perdió relevancia en la nueva matriz de políticas públicas y, como resultado, quedaron subrogadas y estructuralmente 
marginadas y relegadas a la periferia del nuevo esquema de desarrollo económico instituido. Pequeñas y medianas unidades productivas en el sector agrícola pasaron a quedar en una situación de pérdida de condiciones para continuar desarrollando sus actividades productivas tradicionales y locales (Mesalles, 2011).

En definitiva, diversos sectores sociales y productivos pasaron a quedar estructuralmente excluidos del nuevo esquema de desarrollo económico nacional, tanto en zonas rurales como en las urbanas. Sus actividades productivas perdieron interés en el marco de las políticas públicas, y quedaron desvalorizadas y relegadas a sobrevivir sin apoyo y sin protección institucional, expuestas a los vaivenes e incertidumbres de un mercado liberalizado y de una economía altamente abierta a la penetración competitiva de productos extranjeros.

Según señala Mesalles (2011), en un ámbito como el de los salarios y los ingresos, en su lugar: "el crecimiento en salarios y en ingresos, producto de la apertura, se ha venido concentrando en un grupo reducido de agentes económicos, dejando por fuera de los beneficios de la apertura a una parte de la población" (p. 106). Así planteado, tal como reconocen las propias voces promotoras y defensoras del nuevo esquema de desarrollo económico, "los beneficios de la apertura" excluyen a diversos sectores sociales y productivos. Y esta situación excluyente, muy probablemente se llegó a ver aún más consolidada, tras la aprobación en 2007 del TLC Centroamérica/Estados Unidos.

Desde una posición de análisis crítico, González (2010) remarca lo siguiente:

El trabajo formal asalariado ha entrado en crisis. Las economías centrales aumentarán la productividad, disminuyendo el número de puestos de trabajo. En ese cuadro agravado por la crisis financiera se intenta justificar la exclusión y la desigualdad como falta de preparación para la competitividad exigida en tiempos de crisis. Los derrotados o miserables del mundo pagan el precio por su incompetencia o por sus opciones personales y colectivas. (pp. 132-133)

La reforma educativa en Costa Rica, emprendida a mediados de la década de 1990, instituida en la "Política educativa hacia el siglo XXI” y vigente al día de hoy, quedó definida en razón de organizar la formación con base en la ameritación de perfiles laborales que involucraba el nuevo esquema de desarrollo económico nacional.

Emergió así la centralidad de una matriz curricular para la empleabilidad, con un marcado énfasis en la capacitación técnica y en el desarrollo de habilidades y destrezas acordes con los nuevos perfiles laborales demandados. La tecnología curricular pasó a incorporar y desarrollar el enfoque por competencias, así como se empezó a posicionar en el discurso educativo, el lenguaje de la competitividad, del emprendedurismo y la innovación. En el marco de la formación educativa, estos tres tópicos se convirtieron de manera privilegiada en la configuración de "ethos" que ha de inculcarse a las nuevas generaciones en el contexto del actual proyecto educativo nacional. Para los sistemas educativos nacionales, entonces:

El problema de la enseñanza ... es lograr que niños y niñas aprendan las definiciones correctas. Los procesos de pensamiento son definidos como consistentes en manipulaciones de nociones abstractas o de cualidades inconexas siempre examinándolas al margen de cualquier contexto histórico o social; dando por sentado que no importa cómo, ni dónde, ni cuándo, ni por quién se generaron. (Torres, 2005, p. 43)

La referida matriz curricular para la empleabilidad es diversa en sus modalidades de inculcación, pero homogénea y unidireccional en lo esencial de su heurística pedagógica, lógica de contenidos y finalidades: matemáticas, ciencias, comprensión lectora y aprendizaje del inglés, para el desarrollo de las habilidades y destrezas que requiere el mercado de trabajo vinculado a las empresas de inversión extranjera directa, las actividades del turismo transnacionalizado, el sector servicios (los conocidos "call centers", por ejemplo) y del sector empresarial nacional cuyas actividades están volcadas hacia el mercado global.

En consecuencia, tal nueva matriz curricular conduce a soslayar y a indiferenciar la naturaleza propia de las necesidades, intereses y expectativas de formación educativa que conciernen a diferentes poblaciones estudiantiles y comunidades del país. En ese contexto, el proyecto educativo instituye, como precepto fundamental, la representación objetivista de que todas las personas pueden y tienen que aprender y desarrollar el mismo dominio de idénticas habilidades y destrezas cognitivas y técnicas, cuanto las mismas 
pautas de identificación actitudinal. Es decir, la matriz curricular para la empleabilidad establece, en rigor, la estandarización de los aprendizajes y de la formación educativa, en razón de preparar perfiles laborales para el mercado de trabajo ya referido.

Lo anterior se sustenta en la publicitación universalizada de una expectativa ideológica que, durante los últimos años han promovido organismos multilaterales como el Banco Mundial, la Organización para la Cooperación y el Desarrollo Económico (OCDE), la Organización Mundial del Comercio (OMC): reorientar la formación que se brinda en los sistemas educativos nacionales de cada país, hacia "el recorte de los contenidos referidos a las ciencias sociales, humanidades y artes; $y$, por el contrario, por reforzar aquellos conocimientos y destrezas que capacitan mejor para encontrar un puesto de trabajo" (Torres, 2010, p. 87).

En Costa Rica, no obstante, según ya se sabe, la promoción de esa expectativa choca contra la realidad de que en las nuevas generaciones empieza y ha empezado a aparecer y a arraigar la frustración, en la medida que, pese a haber realizado estudios incluso universitarios, cada día es más complicado hallar un empleo en el mercado formal de trabajo. Y este sentimiento de frustración parece haber empezado también a colectivizarse y a tener repercusiones en los propios centros educativos.

Sobre todo, en los niveles del III ciclo y la educación diversificada, el ausentismo, abandono, rezago, extra edad, reprobación, fracaso escolar, desmotivación, indiferencia por el estudio, hoy, constituyen expresiones de una realidad educativa cuyas estadísticas indican la existencia de déficits importantes que afectan el desempeño del sistema educativo, cuanto asimismo la calidad de la educación impartida.

Distintas evaluaciones nacionales e internacionales evidencian que muchos estudiantes no logran adquirir las habilidades y
destrezas mínimas requeridas para incorporarse exitosamente en la sociedad del conocimiento. En el caso de las pruebas del
Programa para la Evaluación Internacional de Alumnos (PISA) 2012, las calificaciones obtenidas están muy lejos de las que
muestran los países miembros de la Organización para la Cooperación y el Desarrollo Económicos (OCDE), organización
a la cual Costa Rica busca adherirse. (Programa Estado de la Nación, 2015, p. 125)

En materia de calidad de la educación y desde la óptica de la evaluación oficial, se pueden identificar al menos dos deficiencias principales: a) el estudiantado no está adquiriendo las habilidades y destrezas mínimas requeridas para incorporarse exitosamente en la sociedad del conocimiento; b) la educación costarricense no está logrando alcanzar los estándares de calidad necesarios para incorporarse a un organismo multilateral como lo es la OCDE.

$\mathrm{Al}$ respecto, cabría preguntarse: a) ¿cuáles son, en la óptica de quienes formulan las políticas públicas y evalúan los correspondientes niveles de logro del sistema educativo, las demandas de formación educativa que instituye la sociedad del conocimiento y, en su lugar, a las que el proyecto educativo ha de dar respuesta, a fin de orientar conforme a ellas sus acciones, esfuerzos, programas y oferta de formación?; b) ¿Acaso "los estándares de calidad necesarios" para incorporarse a la OCDE, son en materia educativa los mismos que implican "las habilidades y destrezas mínimas" necesarias para "incorporarse exitosamente en la sociedad del conocimiento?”.

Según el Programa Estado de la Nación (2015), en su Quinto Informe Estado de la Educación:

En el siglo XXI, el desarrollo económico y social de los países depende en gran parte del talento y la capacidad del conjunto de su población para generar y aplicar nuevo conocimiento. En esta compleja era del conocimiento, de la irrupción de las tecnologías en todos los ámbitos del quehacer humano y grandes desafíos globales, resultan especialmente críticas las capacidades de las personas para resolver problemas, comunicarse, colaborar con otros, seleccionar, analizar y saber aplicar nueva información; desarrollar nuevas ideas y poner en marcha emprendimientos innovadores que contribuyan al bien común tanto local como global. Que los estudiantes logren avanzar en el desarrollo de estas habilidades constituye el principal desafío del sistema educativo en el presente siglo. (p. 138)

En la actualidad, tanto la política pública educativa como las instituciones que se encargan de evaluar su ejecución y el estado en que se encuentra determinan, como referencialidad fundamental para el sistema educativo, atender y dar respuesta a los "grandes desafíos globales" (globalización), por medio de una formación educativa que responda a la necesidad de que los contingentes de la fuerza laboral del país 
adquieran el dominio de las habilidades y destrezas requeridas para la incorporación intensiva de los desarrollos tecnológicos al aparato económico/productivo. Este es, por tanto, el nuevo paradigma a que se refiere el texto de la Política Educativa hacia el Siglo XXI, aprobado en 1994 por el Consejo Superior de Educación de Costa Rica.

No hay otro camino más que reconocer la existencia, hoy, en Costa Rica, no solo de una realidad marcada por significativas desigualdades estructurales de acceso a una educación pertinente, significativa y de calidad, sino que, por esto mismo, la situación que se tiene es la de una educación que repercute en la reproducción de esas mismas desigualdades sociales estructurales.

Un país en el que la educación contribuye a profundizar las desigualdades sociales estructurales y, también, a preservar la reproducción intergeneracional de las desigualdades sociales estructurales, cualquier país que sea, afronta una situación de incumplimiento de la justicia y la equidad, que tenderá a ser cada vez más profunda.

El sistema educativo nacional debe proveer y garantizar la educación para el trabajo, pero no exclusivamente para la empleabilidad ni para la empleabilidad en un determinado mercado de trabajo acotado y restringido.

Las condiciones socioeconómicas y los contextos socioculturales, las expectativas y las necesidades concretas de las comunidades, las familias y de la población estudiantil constituyen los parámetros básicos a partir de los cuales ha de regirse cualquier oferta educativa.

A medida que lo anterior se cumpla, la "educación para todos" ya no será más la misma y única educación homogénea, sino precisamente la que se fundamenta en la diversidad pedagógica, didáctica y de contenido, sobre la base de las propias necesidades, condiciones y expectativas de las diferentes comunidades estudiantiles.

Desde una perspectiva de libertad, equidad, inclusión y democracia, la educación ha de contribuir, también, a incidir en los derroteros del proyecto nacional de desarrollo, de manera que en este sean atendidas y se propongan estrategias institucionales dirigidas a enfrentar y a resolver las desigualdades sociales y productivas, hoy prevalecientes a escala estructural, así como a generar los equilibrios económicos que el desarrollo nacional debe garantizar a la sociedad en general.

\section{LA EDUCACIÓN INTERCULTURAL: REALIDADES Y DESAFíos}

La inequidad y exclusión social constituyen una dinámica sociohistórica continua en la población latinoamericana. Los procesos de conformación y consolidación de los Estados nacionales -desde la idea de nación blanca y estandarizada en términos identitarios y étnicos-, han invisibilizado las diferencias y particularidades de diversos grupos de población: indígena, afrodescendiente, entre otros: "La dominación es el requisito de la explotación, y la raza es el más eficaz instrumento de dominación que, asociado a la explotación, sirve como el clasificador universal en el actual patrón mundial de poder capitalista” (Quijano, 2000a, p. 238).

En este sentido, las dinámicas educativas en el plano nacional costarricense se han caracterizado por una variedad de contextos socioculturales que, en el fondo, apelan a la urgencia por una perspectiva educativa acentuada desde la interculturalidad, que más allá de procurar "aglutinar u homogenizar" desde el plano político-institucional, trascienda en el análisis sobre los procesos dinámicos que configuran -o refiguran- la educación.

Por un lado, la negación de aquellas formas o posturas hegemónicas, patriarcales, occidentales, que se han vehiculizado desde los procesos educativos, para legitimar un sistema hegemónico de relaciones de poder. Por otro -y aquí el énfasis propuesto desde el OBSED-, el reconocimiento de aquellos conocimientos y saberes que se construyen desde las distintas dinámicas socioculturales e identitarias de las diversas colectividades existentes en el territorio nacional y regional. 
En el caso costarricense, a la población indígena se le ha posicionado desde una relación desigual, invisibilizando sus formas tradicionales, substituyéndolas desde un discurso institucional-nacional, mediante la satisfacción de necesidades en educación, salud, vivienda u organización política: los procesos de “incorporación y formalización” en el sistema de educación formal, desde mediados de los años cincuenta, cuando se inician las primeras formas de intervención estatal en la zona de Talamanca (Rojas, 2002).

Dicho en clave de "colonialidad", la existencia de un centro de poder que canaliza, delimita y "filtra" las capacidades y significados sentidos y latentes de los diversos actores; que traduce su heterogeneidad y "contradicción", a partir de un patrón estándar que niega el conflicto inherente -dominador vs dominados(Quijano, 2000b) y que borra las fronteras socio-espaciales; es decir, se anula la memoria, la capacidad de agencia de los actores y, con esto, la historia de los pueblos pasa a una re-escritura ajena y exterior: "La distribución de las gentes en las relaciones de poder tiene, en consecuencia, el carácter de procesos de clasificación, des-clasificación y re-clasificación social de una población, es decir de aquella articulada dentro de un patrón societal de poder de larga duración” (Quijano, 2000a, p. 369).

Desde la óptica de una estrategia de "homologación" de lo indígena frente a lo no indígena, el Estado costarricense ha agregado e integrado "forzosamente" a la población indígena a la dinámica nacional, a partir de los procesos de educación: “...la implementación de programas de estudio que no están considerando en la práctica, la lengua materna como inherente en los procesos de aprendizaje...” (Hilje, 2014, p. 102). Por ejemplo, la mayoría de las lecciones se brindan en castellano ${ }^{[1]}$, y no en la lengua materna -como lo estipula el artículo N. ${ }^{\circ} 28$ del Convenio N. ${ }^{\circ} 169$ de la OIT-, sumado a las carencias en el tema de infraestructura, herramientas o material didáctico, uso y acceso a recursos tecnológicos, etc.

Si bien es cierto, el país ha suscrito y ratificado acuerdos de carácter internacional, (Convenio N. ${ }^{0} 169$ de la OIT sobre Pueblos Indígenas y Tribales en países independientes; y la Declaración de las Naciones Unidad sobre los Derechos de los Pueblos Indígenas, ONU, 2007); con los cuales están obligados a velar y garantizar el respeto de los derechos de los pueblos indígenas y donde se destaca el artículo N. ${ }^{\circ} 14$, inciso N. ${ }^{\circ} 1$ de la Declaración (ONU, 2007): "Los pueblos indígenas tienen derecho a establecer y controlar sus sistemas e instituciones docentes que impartan educación en sus propios idiomas, en consonancia con sus métodos culturales de enseñanza y aprendizaje" (p. 22).

Aunado, se destaca lo referido en el artículo N. ${ }^{\circ} 27$ del Convenio N. 169 de la OIT (1989):

Los programas y los servicios de educación destinados a los pueblos interesados deberán desarrollarse y aplicarse en cooperación con éstos a fin de responder a sus necesidades particulares, y deberán abarcar su historia, sus conocimientos y técnicas, sus sistemas de valores y todas sus demás aspiraciones sociales, económicas y culturales... (p. 76-77)

Desde el año 2013, el Gobierno de la República, junto con el Departamento de Educación Intercultural del Ministerio de Educación Pública (MEP), elaboran el Decreto Ejecutivo N. 37801 que busca solventar las carencias desde una perspectiva de educación intercultural, por lo menos desde lo referido al tema indígena: "Promover el diálogo intercultural y las aptitudes que le ayuden a los estudiantes a participar plenamente y en pie de igualdad en la vida de su propia comunidad, en la vida social de los territorios indígenas, de las regiones donde se encuentran y de la comunidad nacional e internacional" (Poder Ejecutivo y Ministerio de Educación Pública, 2013, Artículo N. 2, inciso N.o 4).

En tal sentido, aproximar la educación intercultural, representa un "acercamiento" a la cuestión de la identidad: por un lado como esquema clasificatorio o discriminante socio-histórico y cultural, desde un discurso moderno de una homogenización cultural que termina por subsumir lo particular, generalizándolo para la totalidad de la población, en una suerte de producto finito y acabado, que supuestamente otorga cohesión social; pero por otro -y puesto acá el énfasis- en la identidad como mecanismo que visibiliza la existencia de diferencias entre grupos humanos, mediante la "urgencia" de una política de reconocimiento de las distintas formas de ser o significarse por los grupos humanos; puesto en palabras de Taylor (1996), vislumbrando “...más de cerca la ligazón entre modernidad e identidad” (p. 10). 


\section{RECONOCIENDO LAS TRAYECTORIAS DE LOS SUJETOS ACTORES EN SUS CONTEXTOS}

Las premisas de un proyecto educativo intercultural deben procurar que se garantice y reivindique a los distintos grupos de población, su identidad y sus diversos contextos socioculturales, visibilizados y reconocidos en espacio y tiempo. Es decir, el reconocimiento del componente identitario con que cuentan los grupos posicionados en condición de oprimidos históricamente, para generar procesos disruptivos para la autodeterminación y para generar cambios en las condiciones desiguales que han pautado su existencia.

La educación intercultural debe ser un enfoque dinámico, que se traduce y construye en los colectivos desde sus formas de asimilación, integración y resistencia, sociohistórica y cotidianamente en el escenario nacional. Por ejemplo, desde Long (2007), se plantea acentuar el análisis desde una perspectiva orientada en el actor, es decir, el reconocimiento de la diferencia, más aún, del carácter heterogéneo de la realidad que circunda los contextos, las relaciones sociales y la vida misma de cada actor: la presencia de múltiples y diversos repertorios culturales, intereses, agendas.

Lo que se procura indagar será el cómo interpretan o articulan -desde cada actor- las complejidades que derivan del encuentro entre "mundos de vida diferenciados y los procesos de construcción cultural" (Long, 2007, p. 110); es decir, cuáles son las formas en que las distintas poblaciones procesan la idea -en este caso- de educación y sus posibles significados desde su experiencia; pero donde siempre existe la posibilidad de generar alternativas para disentir o desentrañar "acomodamientos comunes": “...se pretende entender la producción de fenómenos culturales heterogéneos y los resultados de la interacción entre dominios de representación y discursivos, y así delinear lo que podríamos llamar una cartografía de diferencia cultural, poder y autoridad" (Long, 2007, p. 110).

El primer paso debe implicar el que se "rechace un concepto de cultura homogéneo" (Long, 2007, pp. 111-112), que puede llevar a juicios valorativos que encasillan la experiencia de los actores: por ejemplo, la idea errónea que a los grupos indígenas se les debe educar porque su condición de atraso o escaso desarrollo les otorga una ignorancia innata. Más bien, sugiere la consideración de tres categorías de análisis que pueden contribuir en el proceso: por un lado, el de repertorios culturales, referenciado a los diversos elementos culturales (nociones de valor, símbolos, procedimientos rituales, etc.) que utilizan en la práctica social. Por otro lado, la heterogeneidad, reconociendo la coexistencia formas sociales diversas en un escenario cultural particular. Por último, el hibridismo, que sugiere la combinatoria posible de repertorios culturales distintos, que son confrontados, negociados o consensuados de manera estratégica, por los sujetos actores involucrados.

En esta línea, se reivindica la no negación del conflicto, sino más bien se reconoce y destaca su carácter dialéctico y fuente de análisis, desde los actores y significados en disputa y para ello. Esto es, reconocer al actor en su capacidad de agencia: “...capacidad de saber y capacidad de actuar...” (Long, 2007, p. 48). No será únicamente la satisfacción de las necesidades -desde el Estado-, sino también la confrontación de demandas, en un escenario de relación vis-á-vis con los otros grupos -desde la población indígena-. En resumidas cuentas: una educación intercultural que se construye colectivamente.

\section{LA RESISTENCIA Y SUS FORMAS ESCENIFICADAS: CONSTRUYENDO EDUCACióN INTERCULTURAL}

La educación, en sentido estricto, se asume como una práctica política, que se redefine cotidianamente en el diálogo de saberes e ignorancias, entre los actores situados o contextualizados: un espacio donde se estructuran posiciones ocupadas por diversos agentes que, en virtud de sus posiciones, tienen un espacio de luchas: dominador y dominados, entre lo moderno y lo tradicional, entre lo urbano y lo rural; desde el caso particular que se ha venido referenciando, desde lo indígena y lo no indígena: las formas en que asimilan o suponen se integran al plano nacional desde lo educativo, que devienen en prácticas excluyentes. Pero 
donde también -siguiendo la perspectiva del actor- la población indígena, re-traduce o bien re-significa las necesidades, para sobrevivir y en tanto, resistir.

Más allá de una suerte de tecnicismos formales, el abordaje de una educación intercultural debe partir considerando que las situaciones de vida de los actores inmersos, se caracterizan por una constante fuente de tensión, desde un análisis minucioso en el dónde y en el cómo construyen sus mundos de vida, los cuales contienen/evidencian mecanismos diversos de resistencia:

Luchas de ideas, valores, intereses y sentimientos que se reflejan como puntos concretos de consenso. La gente, al disentir o consentir, pueden estar conectándose o desconectándose de las diversas redes sociales y cambiar el sentido de sus alianzas políticas o lealtades... Lo central es analizar cómo se incluyen o excluyen intereses e iniciativas que enrolan a la gente en la creación de su espacio de maniobra y sus proyectos de vida”. (Torres, 1997, p. 190)

Esto es, hacer patente la configuración dinámica de la educación a partir de las subjetividades y del reconocimiento de las diferencias socioculturales. El reto está en términos de cuáles deberían ser las condiciones y dimensiones mínimas, con las que debería contar una propuesta de educación intercultural ${ }^{[2]}$ , que procure subsanar las dinámicas de exclusión sobre la población indígena y otros colectivos; pero, sobre todo, que en la práctica atienda de forma fehaciente la realidad inmersa en torno al tema educativo, su acceso e incidencia en la población y desde esta, y en sus espacios situados y significados.

\section{LA EVALUACIÓN EN LA EDUCACIÓN: ¿DESDE QUÉ PERSPECTIVA EVALUAR?}

Este apartado está dirigido a proponer una visión de la disciplina evaluativa, en términos de superar el enfoque de la rendición de cuentas como un ejercicio obligatorio de las instituciones del Estado, para fundamentar la evaluación como una referencialidad conceptual y programática que permite la mejora de la educación y de los sistemas educativos, en razón de la excelencia académica y de la transformación educativa para el logro de una auténtica equidad y para una educación con sentido concreto de justicia social.

La finalidad es colocar, en contexto, la disciplina evaluativa, en asociación íntima con los propósitos y los requerimientos de una educación socialmente comprometida; relevar la dimensión social de la educación.

La evaluación de los sistemas educativos, en Costa Rica como en otros países, ha pasado a ser ahora una práctica bastante institucionalizada, cuyo enfoque e instrumentación presentan una lógica estandarizada y tecnocratizada. Contrario a esta noción, se propone aquí un enfoque de evaluación de alcance comprensivo, pluralista y multidimensional.

Murillo y Román (2010) sostienen que, en América Latina, en términos generales, predominan perspectivas de evaluación que analizan componentes disgregados y compartimentalizados. En contrapropuesta, estos analistas anclan en proponer una evaluación que acuda a la integralidad de los procesos y a la interdependencia de las acciones, las políticas y los actores. A su juicio:

La evaluación ha de contribuir a la mejora de los niveles de inclusividad de la educación, facilitando la construcción de estrategias para que todos puedan desarrollarse plenamente a partir de sus capacidades, saberes previos, intereses y necesidades. La justicia educativa y su calidad también se deberán medir por la capacidad de los sistemas escolares de incorporar y potenciar a todos los niños y jóvenes. (Murillo y Román, 2010, p.106)

Frente a la disgregación de componentes y con la mira puesta en la incorporación de diversos agentes de los sistemas escolares, nos adherimos a la propuesta de evaluación para la transformación, que Vargas (2001) define como: "un proceso de autorreflexión y de producción de conocimiento que realizan las y los [sic] participantes sobre la teoría y la práctica de su propia acción” (p. 10).

Si se parte de esta definición, la evaluación asume una práctica que trasciende la descripción y la caracterización para promover cambios en lo evaluado y en las personas, así como para sustentar una perspectiva de reconstrucción teórica del campo y de aportación sustantiva a este. 
Los cambios que se buscan parten de la proposición, según la cual, el elemento a evaluar puede transformarse y la evaluación contribuye a que, en su contexto inmediato, se den transformaciones tangibles, las que en el futuro podrán ser convertidas en transformaciones de mayor impacto.

La consideración de este enfoque, que releva la diversidad de actores y agentes educativos, busca facilitar y potenciar la participación de estos grupos y garantizar su protagonismo. Esta visión y ruta de evaluación es acorde con lo que se denomina como "evaluación pluralista", contrapuesta "a la idea de evaluación 'imparcial' y 'objetiva'. Desde esta posición, las aproximaciones pluralistas se convierten en evaluaciones 'sensibles' a los intereses de los actores que participan en la evaluación” (Izquierdo, 2008, p. 116).

El punto de partida es, entonces, que la tarea de evaluar programas, proyectos o políticas educativas deviene en una consideración de los distintos participantes/agentes: sean entes destinatarios, decisores, ejecutores o sociedad civil en general; por cuanto la educación, en su dimensión social, penetra instituciones, estructuras sociales, acciones-interacciones y personas así como poderes e intereses.

Es pertinente referir la indicación hecha por House (1997), en el sentido de que "la evaluación aspira a persuadir a un público concreto del valor de algo" (p. 71).

[Tal acción persuasiva] ... está directamente relacionada con la acción. Aunque la información de la evaluación no sea tan segura como la información científica dirigida a una audiencia universal, la persuasión es eficaz para promover la acción porque se centra en un público concreto y suministra la información que afecta a ese público. (House, 1997, p. 72)

Este ejercicio persuasivo de la evaluación coincide con la consideración de múltiples audiencias y la distribución del poder e intereses existentes, así como con la desigual participación y capacidad de reacción ante resultados de evaluación.

Otra finalidad insoslayable en todo ejercicio evaluativo es la de delinear medidas, estrategias o decisiones para mejorar. En esta intencionalidad, la precisión y delimitación de las alternativas posibles es crucial. En este propósito evaluativo es factible incidir en el curso de programas, proyectos y políticas cuando, según los resultados de las valoraciones obtenidas, se sugieren rumbos a seguir; es decir, cuando se adhieren las recomendaciones para la elaboración y desarrollo de nuevas programaciones.

La educación es un amplio campo de indagación evaluativa, en tanto coexisten en ella, como en otros campos de conocimiento, diversidad de posicionamientos epistémicos, perspectivas teóricas y estrategias metodológicas. Es esta la razón por la que se asume aquí una perspectiva amplia, pluralista, múltiple y participativa, acerca de la evaluación en educación: la perspectiva de la evaluación para transformar y mejorar.

\section{EVALUACIÓN TRANSFORMADORA: SUPERAR LA DICOTOMÍA CUANTITATIVA Y CUALITATIVA}

La evaluación como campo disciplinar se nutre de distintos enfoques, entre los que cabe mencionar el de la praxis crítica, orientado hacia la transformación de lo social con la finalidad de mejorar las condiciones de la educación y del quehacer educativo. Este enfoque ha transitado por diferentes propuestas teórico/prácticas, originadas desde diferentes nociones de la realidad, influenciadas por las estructuras política, económica y cultural.

Según Vargas (2001), al estar las ideas neoliberales difundidas por el orbe, se crean políticas con tendencias a reducir el financiamiento para la educación, limitantes de la participación estatal, favorecedoras de procesos de privatización y enfocadas en fortalecer la educación técnica con tendencias a la profesionalización, en respuesta a las demandas de las economías globales y del mercado. Al respecto, Muñoz (2015) subraya que esa orientación involucra un proyecto de formación centrado fundamentalmente en la habilitación educativa para la empleabilidad: "en la educación estatal costarricense ha regido... la que privilegia la formación educativa en referencia directa, orga\#nica y siste\#mica con su adecuacio\# $\mathrm{n}$ a los requerimientos del desarrollo econo\#mico y, como en la actualidad acontece cada vez ma\#s, la formacio\#n y capacitacio\#n para el mercado de trabajo y la empleabilidad (p. 2). 
En el contexto de dichas dinámicas político/económicas es donde se desarrollan las primeras tendencias evaluativas en educación. Según Díaz (1993), el examen como tal, es un punto de partida importante, ya que este se sitúa en el centro de la política educativa de corte neoliberal; política que pretende una reducción presupuestaria en la educación, limitando el acceso a esta por medio de diversos mecanismos, entre los cuales el examen estandarizado cuantitativo cumple una función fundamental.

Todo el mundo sabe que el examen es el instrumento a partir del cual se reconoce administrativamente un conocimiento, pero igualmente que el examen no indica realmente cuál es el saber del sujeto. (Díaz, 1993, p. 129)

El examen, como medición de la calidad, eficiencia y eficacia de los aprendizajes, se populariza, consolida y reproduce en las políticas de los procesos globalizados de corte mercantilista, que inducen a realizar recortes de presupuesto, además de instrumentalizar la descentralización y la autonomía escolar (Tiana, 2009), restándole responsabilidad al Estado. Así, las pretensiones de la evaluación se hacen evidentes, en cuanto se pretende que esta funja como mecanismo catalizador que liga los resultados con el financiamiento, la rendición de cuentas, la calidad y su vinculación con la empresa (Vargas, 2001).

La evaluación de los aprendizajes parte de la premisa, sustentada en la sociología positivista ${ }^{[3]}$, de que la medición de conocimientos, habilidades y atributos en las personas estudiantes ha de estar orientada a la toma de decisiones correctivas sobre el futuro educativo de la niñez, la adolescencia y la juventud estudiantil. Para ello se crean instrumentos especialmente cuantitativos y estandarizados, basados en perspectivas sobre la validez y la confiabilidad de la evaluación provenientes de la psicometría (De Alba, 1991; Díaz, 1993; Vargas, 2001). Desde estos postulados surgen diferentes corrientes evaluativas, en las que se desarrollan estándares para conocer si lo evaluado consigue lo planteado y pretendido en los objetivos.

En una línea que introduce lo cualitativo a la par de lo cuantitativo nace, por su parte, la evaluación sistémica, pensada como un proceso integral, consustancial de la función educativa. Esta perspectiva de la evaluación pretende conocer, realimentar y generar una mejora en el sistema educativo y en sus subsistemas, pensándola como una evaluación más integral (Vargas, 2001). No obstante, esta perspectiva no logra superar el dualismo objeto-sujeto.

Estas tendencias coinciden en un enfoque positivista de lo educativo, que pretende que la realidad educativa sea "predecible, medible, cuantificable y controlable y que es posible parcelar el conocimiento de esa realidad mediante diferentes evaluaciones que analizan elementos de esa realidad educativa en forma descontextualizada y generalizada" (Vargas, 2001, p. 6). No se toma en cuenta el contexto, la situación socio histórica en la que se da el fenómeno educativo, ya que ponen el énfasis en el control y la medición instrumental/objetivista, que pasan a implicar situaciones de dominio por medio de poder institucional hegemonizante (De Alba, 1991; Díaz, 1993; Vargas, 2001), en las cuales se favorece el desarrollo de un currículum estandarizado que responde a los intereses educativos interpuestos por los sectores sociales y económicos dominantes y hegemónicos en la estructura del sistema de relaciones de poder.

Por su parte, las propuestas que trascienden el enfoque positivista ${ }^{[4]}$ proporcionan un cambio clave en la concepción del papel y lugar de la subjetividad en la evaluación, colocándola como un eje articulador del proceso holístico en el que la evaluación debe llevarse a cabo. Se parte de una perspectiva en la que las realidades educativas son entendidas como construcciones sociales. Esto implica que se resignifique la interacción de subjetividad que media entre quien observa y lo observado, se busca llegar con ello a un consenso entre todas las partes involucradas en el proceso evaluativo, incluye a quien evalúa y que está a cargo de construir las realidades de diferentes personas en referencia a lo evaluado (Vargas, 2001).

Aunque estos modelos parten de un paradigma diferente y pretenden llegar a lo comprensivo, se centran en la dinámica interna de lo institucional, dejando por fuera el análisis de las fuerzas sociopolíticas, económicas y culturales que también son parte de lo evaluado y necesarias para poder llegar a la transformación.

Con la finalidad de lograr la comprensión y la valoración, De Alba (1991) plantea lo siguiente: 
En el proceso de evaluación se da un interjuego de comprensión y valoración, en el cual se encuentran instituidos o se instituyen los códigos de evaluación (análisis conceptual, valoración axiológica), los cuales en un momento dado pueden ser a su vez revisados, reinstituidos. Es importante retomar de esta corriente el carácter dinámico tanto de los procesos sociales como de las instituciones que en éstos se conforman, con la intención de recuperar el carácter activo de los sujetos sociales involucrados en la evaluación. (p. 92)

En el marco de la evaluación posicionada en los modelos transformativos que nacen en el contexto de la pedagogía crítica, "surgen nuevas alternativas para la evaluación que tratan de explicar la realidad social analizando la dicotomía teoría-praxis y sujeto-objeto, en la acción social e incorporando la perspectiva del currículum como una forma de política cultural" (Vargas, 2001, p. 9). Para decirlo en términos de Foucault (1976), lo que se busca es abrir, en los espacios educativos, los lugares que permitan rescatar las voces de los grupos acallados.

Dentro de la pedagogía crítica, la evaluación busca consolidarse como un proceso de autorreflexión y de producción de conocimiento que realizan quienes participan de la propia acción evaluativa. La evaluación "se convierte así en praxis crítica a lo largo de todo el proceso educativo, pero su interés no es únicamente reflexionar sino promover cambios, tanto en las personas como en lo evaluado" (Vargas, 2001, p. 10).

La evaluación transformadora supera la dicotomía sujeto-objeto, ya que, además de centrarse en la condición de la subjetividad humana, se enmarca en la dialéctica social, política y económica de los procesos educativos. A la vez que toma en cuenta el lugar del poder y sus procesos hegemónicos, para superarlos y transformarlos en concordancia con el contexto y sus particularidades.

El enfoque transformador se sustenta en la epistemología (modo de conocer) de la totalidad, y desde ahí la evaluación requiere visualizar la historicidad del proceso mismo (su origen, su desarrollo, su devenir). A la par, es necesario analizar los elementos estructurales que han permanecido relativamente estables, así como la función que cumplen en el desarrollo y consolidación de ciertas tendencias, problemáticas, expresiones, marginaciones o inhibiciones que se evidencian en la acción educativa. (De Alba, 1991, p. 11)

Sumado a esto, destaca el manejo ético de la producción de la información cuando se evalúa, así como la transparencia a la hora de elaborar los informes y realizar la presentación de los hallazgos. Es importante acotar que dichas disposiciones son parte de la perspectiva epistemológica de la totalidad, ya que estas permiten reconstruir espacios en donde todo relato es tomado en cuenta, a partir de la construcción de aquellos que no han sido visibilizados.

Importa mucho la concepción que de la evaluación se tenga. De ella dependerá, obviamente, la evaluación que se haga. Hay realidades educativas de extrema complejidad que no pueden ser abordadas por procedimientos simplificados. Unos métodos estereotipados no pueden recoger la riqueza de la actividad educativa. (Santos - Guerra, 2000, p. 1)

En síntesis, para arribar a la transformación, el proceso evaluativo se conforma por una complejidad que entrama todas las dimensiones educativas, por lo tanto, para poder lograr la transformación es necesario rescatarlas todas, junto con lo histórico, lo axiológico y lo significante del entramado de relaciones de lo evaluado (De Alba, 1991).

\section{A MANera DE CIERRE}

El contexto nacional y regional de la educación exige y convoca la reflexión sobre la experiencia desarrollada por el equipo del OBSED, que considera la lectura de las necesidades y las experiencias de investigación. En los espacios, en las vinculaciones que se originan entre actores académicos y sociales y con estos, emergen permanentemente los desafíos, problemáticas, incertidumbres e interrogantes que es necesario enfrentar como Observatorio de la Educación y que se encuentran en los apartados anteriores. Se tiene la convicción de que, de este modo, la producción de conocimiento tiene una legitimidad amplia, tanto en la academia como en la sociedad. 
Interpela al OBSED la preocupación por trabajar hacia una educación que reconozca y releve las necesidades y expectativas de realidades socioculturales diversas de la sociedad nacional y regional. Se ha optado por ubicarse en un horizonte abierto, en torno a temas, hechos, situaciones, modelos y sistemas en educación e investigación. El horizonte del equipo del OBSED se dibuja, encuentra sus trazos día a día, a través de la lectura y los debates sobre los procesos socio-educativos actuales en América Latina y El Caribe.

Contribuye al fortalecimiento del OBSED pensarse en la posición de sujeto colectivo cognoscente crítico, propositivo y sensible a los cambios, hacer periódicamente una reflexión epistémica desde las interrogantes que emergen de los procesos, sujetos, subjetividades y aproximaciones metodológicas, que articulan la calidad de la investigación con las variadas formas de conocimiento de cada persona investigadora.

La mirada del OBSED se construye en la práctica de observación. Surge en un presente activo y potencial. Esa mirada no tiene, desde la perspectiva epistémica, un carácter simplemente evaluativo. Sostiene la legitimidad de un proceso reflexivo abierto a los cambios y con una construcción constante de la maduración intelectual, académica, espiritual y afectiva del equipo, que rompe con las especialidades y exclusividades del tratamiento de conocimientos; que supera las soledades comúnmente presentes en la tarea investigativa, para apoyarse en la disposición de escuchar voces, diferir, compartir y encauzar una búsqueda de manera colectiva y colaborativa.

Los apartados temáticos incluidos en este artículo representan la trama de preocupaciones, dilemas y ejes de acción investigativa, relacionados con la situación de la educación y del proyecto educativo nacional, que es expresión de la relación sistémica y programática con la cultura, lo económico-productivo y la institucionalidad del país, asumida y legitimada de una determinada manera.

\section{ReFERENCIAS}

Coordinación Educativa y Cultural Centroamericana, Sistema de la Integración Centroamericana. (2013). Política Educativa Centroamericana 2013-21. Recuperado de https://issuu.com/ccesvelsalvador/docs/ pec_aprobada_7_dic_13

Consejo Superior de Educación. (1994). Política Educativa hacia el Siglo XXI. San José, Costa Rica: Ministerio de Educación Pública. Recuperado de http://www.cse.go.cr/actas/politica-educativa-hacia-el-siglo-xxi

De Alba, A. (1991). Evaluación curricular. Conformación conceptual del campo. México DF: Universidad Nacional Autónoma de México.

Díaz, A. (1993). El examen: Un problema de historia y sociedad. En A. Díaz (Coord.), El examen: textos para su historia y debate (pp. 11-28). México D.F.: UNAM.

Foucault, M. (1976). Historia de la locura en la época clásica. México: Fondo de Cultura Económica.

González, M. (2014). Los procesos de transformación de la política educativa en Centroamérica y República Dominicana desde una visión de la educación comparada (1990-2012) (Informe final de investigación). Instituto de Investigación en Educación. Universidad de Costa Rica. Costa Rica.

González, M. (2010). Los colectivos depauperados repolitizan los currícula. En J. Gimeno (Comp.). Saberes e incertidumbres sobre el currículum (pp. 128-148). Madrid: Ediciones Morata.

Hilje, W. (2014). La refiguración cultural indígena en Costa Rica: El caso de los bribri (Talamanca, provincia de Limón, Costa Rica) (Tesis de maestría). FLACSO, México.

House, E. (1997). Evaluación, ética y poder (2da ed.). Madrid: Ediciones Morata.

Izquierdo, B. (2008). De la evaluación clásica a la evaluación pluralista. Criterios para identificar los distintos tipos de evaluación. EMPIRIA. Revista de Metodología de Ciencias Sociales, 16, 115-134. Recuperado de www.redalyc.org/pdf/2971/297124024005.pdf

Long, N. (2007). Sociología del desarrollo: Una perspectiva centrada en el actor. México: Centro de Investigaciones y Estudios Superiores en Antropología Social. Colegio de San Luis, México. 
Mesalles, L. (2011). Costa Rica: Resultados de la apertura económica. En L. Mesalles y A. Pacheco (Eds.), Costa Rica y su modelo de crecimiento: Revisión a la luz de la crisis internacional. Academia de Centroamérica (pp. 67-114). Recuperado de http://www.hacienda.go.cr/cifh/sidovih/uploads/archivos/Libro/Costa\%20rica\%20y \%20su\%20medelo\%20de\%20crecimiento-libro.pdf

Muñoz, L. (2015). Educación y desarrollo económico en Costa Rica. Apuntes para la discusión. Observatorio de la Educación Nacional y Regional. Recuperado de http://observatorio.inie.ucr.ac.cr/

Murillo, J. y Román, M. (2010). Retos en la evaluación de la calidad de la educación en América Latina. Revista Iberoamericana de Educación 53, 97-120. Recuperado de http://rieoei.org/rie53a05.html

Organización de Naciones Unidas (ONU). (2007). Declaración sobre los derechos de los pueblos indígenas. Recuperado de http://www.un.org/esa/socdev/unpfii/documents/DRIPS_es.pdf

Organización Internacional del Trabajo (OIT). (1989). Convenio N. ${ }^{\circ} 169$ sobre pueblos indígenas y tribales en países independientes. Cuadernos de Legislación Indígena. México DF: Comisión Nacional para el Desarrollo de los Pueblos Indígenas. Recuperado de http://www.cdi.gob.mx/transparencia/convenio169_oit.pdf

Poder Ejecutivo y Ministerio de Educación Pública. (2013). Reforma del subsistema de educación indígena. Decreto N. ${ }^{\circ}$ 37801. Diario Oficial La Gaceta, 135. Recuperado de http://www.gaceta.go.cr/pub/2013/07/15/ COMP_15_07_2013.html\#_Toc361412525

Programa Estado de la Nación. (2015). Quinto Informe Estado de la Educación. San José, Costa Rica: Programa Estado de la Nación. Recuperado de http://www.estadonacion.or.cr/educacion2015/assets/cap-3-ee-2015.pdf

Quijano, A. (2000a). Colonialidad del poder, eurocentrismo y América Latina. En La colonialidad del saber: Eurocentrismo y ciencias sociales. Perspectivas Latinoamericanas. E. Lander. Buenos Aires: CLACSO. Recuperado de http://bibliotecavirtual.clacso.org.ar/clacso/sur-sur/20100708034410/lander.pdf

Quijano, A. (2000b). Colonialidad del poder y clasificación social. Journal of World-Systems Research, 11(2), 1-33. Recuperado de http://bibliotecavirtual.clacso.org.ar/clacso/sur-sur/20100708034410/lander.pdf

Rojas, D. (2002). El conflicto entre tradición y modernidad: Constitución de la identidad cultural indígena bribri. Cuadernos de Ciencias Sociales 126, 7-57. San José, Costa Rica: FLACSO. Recuperado de http:// unpan1.un.org/intradoc/groups/public/documents/icap/unpan045141.pdf

Santos-Guerra,J. (2000). La evaluación: Un proceso de diálogo, comprensión y mejora. Málaga: Universidad de la Salle.

Taylor, Ch. (1996). Fuentes del yo: La construcción de la identidad moderna. Barcelona, España: Paidós.

Tiana, A. (2009). Evaluación y cambio educativo: Los debates actuales sobre las ventajas y los riesgos de la evaluación. En E. Martín y F. Martínez (Coords.), Avances y desafíos en la evaluación educativa (pp. 17-26). Madrid, España: Fundación Santillana.

Torres, J. (2005). El curriculum oculto. Madrid: Ediciones Morata.

Torres, J. (2010). Currículum, justicia e inclusión. En J. Gimeno (Comp.), Saberes e incertidumbres sobre el currículum (pp. 84-102). Madrid: Ediciones Morata.

Torres, G. (1997). La fuerza de la ironía. Un estudio del poder en la vida cotidiana de los trabajadores tomateros de occidente de México. Jalisco: El Colegio de Jalisco- CIESAS.

Valerión J. y Esquivel, J. M. (2006). Situación de la evaluación y la investigación educativa en los países centroamericanos y la República Dominicana. San José, Costa Rica: Coordinación Educativa y Cultural Centroamericana (CECC) y Agencia Española de Cooperación Internacional (AECI). Recuperado de http:// unpan1.un.org/intradoc/groups/public/documents/icap/unpan038669.pdf

Vargas, A. (2001). Enfoques evaluativos. Revista de Ciencias Sociales, 2-3(93), 35-45. Recuperado de http:// www.redalyc.org/pdf/153/15309304.pdf

\section{Notas}

[1] En los centros educativos en zona indígena, sus docentes deben necesariamente ser o pertenecer al grupo indígena; sin embargo, los contenidos de las lecciones se deben de ajustar a lo estipulado institucionalmente en los planes de estudio y 
currículos a nivel nacional: el tema de la lengua materna, se "subsana” con una clase "aparte y específica” con la modalidad de un "docente de lengua indígena".

[2] Con esto, no se afirma que no existan anteriores experiencias que han aproximado interculturalmente a la educación, sino más bien se procura destacar la relevancia de la temática como parte de los desafíos en el quehacer desde el OBSED.

[3] Los primeros aportes a la disciplina evaluativa, que inicia a principios del siglo XX, parten de la sociología positivista o empirista, la cual pretendía explicar los hechos sociales, prediciendo su comportamiento e intentando desarrollar conocimiento objetivo de la realidad (Vargas, 2001).

[4] Dentro de las propuestas que trascienden el enfoque positivista, se encuentran el modelo iluminativo (Parlett y Hamilton), la evaluación respondente (Stake), la hermenéutica constructivista (Guba y Lincoln), y la democrática (House y Kowe); el aporte que realizan estos modelos se basa en entender la evaluación como un proceso complejo y dinámico; tienen en cuenta que en la evaluación, como proceso, influyen dinámicas generadas entre lo que se evalúa, el sujeto evaluador y los otros actores que intervienen en el proceso (Vargas, 2001).

\section{BY-NC-ND}

